\title{
Article \\ Estimates for a Rough Fractional Integral Operator and Its Commutators on $p$-Adic Central Morrey Spaces
}

\author{
Naqash Sarfraz ${ }^{1}$ (D) and Fahd Jarad $2,3, *$ (D) \\ 1 Department of Mathematics, University of Kotli Azad Jammu and Kashmir, Kotli 11100, Pakistan; \\ nsarfraz@math.qau.edu.pk \\ 2 Department of Mathematics, Çankaya University, Ankara 06790, Turkey \\ 3 Department of Medical Research, China Medical University Hospital, China Medical University, \\ Taichung 40402, Taiwan \\ * Correspondence: fahd@cankaya.edu.tr
}

check for

updates

Citation: Sarfraz, N.; Jarad, F. Estimates for a Rough Fractional Integral Operator and Its

Commutators on $p$-Adic Central Morrey Spaces. Fractal Fract. 2022, 6, 117. https://doi.org/10.3390/ fractalfract6020117

Academic Editors: Hijaz Ahmad and Haibo Chen

Received: 27 December 2021 Accepted: 15 February 2022 Published: 16 February 2022

Publisher's Note: MDPI stays neutral with regard to jurisdictional claims in published maps and institutional affiliations.

Copyright: (C) 2022 by the authors. Licensee MDPI, Basel, Switzerland. This article is an open access article distributed under the terms and conditions of the Creative Commons Attribution (CC BY) license (https:// creativecommons.org/licenses/by/ $4.0 /)$.

\begin{abstract}
In the current paper, we obtain the boundedness of a rough $p$-adic fractional integral operator on $p$-adic central Morrey spaces. Moreover, we establish the $\lambda$-central bounded mean oscillations estimate for commutators of a rough $p$-adic fractional integral operator on $p$-adic central Morrey spaces.
\end{abstract}

Keywords: central Morrey spaces; commutators; rough $p$-adic fractional integral operator; $\lambda$-central boundedned mean oscillations

MSC: 42B20; 42B25

\section{Introduction}

In this day and age, fractional calculus is a key area because of its heaps of applications in engineering science and technology, see for instance [1,2]. Moreover, fractional integral operators are major part of the mathematical analysis. These operators have been used to formulate and construct new results in the theory of inequalities. Many of the familiar inequalities and relevant results are generalized and extended via fractional integral operators $[3,4]$. Fractional integral operator of order $\beta$ is defined by

$$
T_{\beta} f(x)=\frac{1}{\zeta_{n}(\beta)} \int_{\mathbb{R}^{n}} \frac{f(\mathbf{y})}{|\mathbf{x}-\mathbf{y}|^{n-\beta}} d \mathbf{y},
$$

where $\zeta_{n}(\beta)=\frac{\pi^{n / 2} 2^{\beta} \Gamma(\beta / 2)}{\Gamma((n-\beta) / 2)}$. A fractional integral operator is a smooth operator and has been applied in several branches such as partial differential equations, harmonic analysis, non-linear control theory, and potential analysis, see for example [5,6] and references therein. Over the years, the boundedness properties of $T_{\beta}$ has put many researchers in the spotlight [7-9].

In the last few years. the field of $p$-adic numbers $\mathbb{Q}_{p}$ is wildly used in harmonic analysis [10-12] and mathematical physics $[13,14]$. Let $p$ be a prime number. The field of the $p$-adic absolute value $|y|_{p}$ is defined by setting $|0|_{p}=0$,

$$
|y|_{p}=p^{-\gamma} \quad \text { if } \quad y=p^{\gamma} \frac{s}{t}
$$

where $\gamma, s, t \in \mathbb{Z}$, and $p, s$ and $t$ are coprime. $|\cdot|_{p}$ undergoes many axioms of a real norm with the below ultrametric inequality

$$
|y+z|_{p} \leq \max \left\{|y|_{p},|z|_{p}\right\}
$$


In [14], we see that any $y \neq 0 \in \mathbb{Q}_{p}$ can be uniquely represented as:

$$
y=p^{\gamma} \sum_{i=0}^{\infty} \alpha_{i} p^{i}
$$

where $\alpha_{i}, \gamma \in \mathbb{Z}, \alpha_{i} \in \frac{\mathbb{Z}}{p \mathbb{Z}_{p}}, \alpha_{0} \neq 0$. The convergent of the series (3) is from $\left|p^{\gamma} \alpha_{i} p^{i}\right|_{p}=p^{-\gamma-i}$.

The space $\mathbb{Q}_{p}^{n}$ consists all $n$-tuples of $\mathbb{Q}_{p}$ with the following norm

$$
|\mathbf{y}|_{p}=\max _{1 \leq k \leq n}\left|y_{k}\right|_{p}
$$

Now, let

$$
B_{\gamma}(\mathbf{a})=\left\{\mathbf{y} \in \mathbb{Q}_{p}^{n}:|\mathbf{y}-\mathbf{a}|_{p} \leq p^{\gamma}\right\}, S_{\gamma}(\mathbf{a})=\left\{\mathbf{y} \in \mathbb{Q}_{p}^{n}:|\mathbf{y}-\mathbf{a}|_{p}=p^{\gamma}\right\}
$$

be, respectively, the ball and sphere with radius $p^{\gamma}$ and the center at a.

It is a familiar fact that $\mathbb{Q}_{p}^{n}$ is a locally compact commutative group under addition; denote by $d \mathbf{y}$, the Haar measure on $\mathbb{Q}_{p}^{n}$ normalized by $\int_{B_{0}(\mathbf{0})} d \mathbf{y}=1$. Additionally, $\int_{B_{\gamma}(\mathbf{a})} d \mathbf{y}=p^{n \gamma}$ and $\int_{S_{\gamma}(\mathbf{a})} d \mathbf{y}=p^{n \gamma}\left(1-p^{-n}\right)$, for any $\mathbf{a} \in \mathbb{Q}_{p}^{n}$.

Suppose $L^{r}\left(\mathbb{Q}_{p}^{n}\right)(1 \leq r<\infty)$ is the space of all complex-valued functions $f$ on $\mathbb{Q}_{p}^{n}$ such that

$$
\|f\|_{L^{r}\left(\mathbb{Q}_{p}^{n}\right)}=\left(\int_{\mathbb{Q}_{p}^{n}}|f(\mathbf{y})|^{r} d \mathbf{y}\right)^{1 / r}<\infty .
$$

In [15], author introduced the fractional integral operator on $\mathbb{Q}_{p}^{n}$ as

$$
T_{\beta}^{p} f(\mathbf{x})=\frac{1}{\Gamma_{n}(\beta)} \int_{\mathbb{Q}_{p}^{n}} \frac{f(\mathbf{y})}{|\mathbf{x}-\mathbf{y}|_{p}^{n-\beta}}, \quad 0<\beta<n,
$$

where $\Gamma_{n}(\beta)=\frac{1-p^{\beta-n}}{1-p^{-\beta}}$.

The explicit formula of the above operator on the $p$-adic field is acquired in $[16,17]$. The fundamental properties of the fractional integral operator on local fields are given in [15]. Moreover, $\lambda$ central bounded mean oscillations estimate for commutators of fractional integral operator on $p$-adic Morrey spaces are reported in [18]. Recently, the boundedness of the fractional integral operator on Morrey spaces is shown in $[12,19]$. The current paper deals with the roughness of an operator which is a key topic in analysis in this day and age; see for instance $[20,21]$ and the references therein. Motivated by [21], we define the rough fractional integral operator. Suppose $b: \mathbb{Q}_{p}^{n} \rightarrow \mathbb{R}, f: \mathbb{Q}_{p}^{n} \rightarrow \mathbb{R}$ and $\Omega: S_{0} \rightarrow \mathbb{R}$ are measurable mappings, then

$$
T_{\beta, \Omega}^{p} f(\mathbf{x})=\int_{\mathbb{Q}_{p}^{n}} \frac{\Omega\left(|\mathbf{y}|_{p} \mathbf{y}\right) f(\mathbf{y})}{|\mathbf{x}-\mathbf{y}|_{p}^{n-\beta}} d \mathbf{y}
$$

and

$$
T_{\beta, \Omega}^{p, b} f(\mathbf{x})=\int_{\mathbb{Q}_{p}^{n}} \frac{(b(\mathbf{x})-b(\mathbf{y})) \Omega\left(|\mathbf{y}|_{p} \mathbf{y}\right) f(\mathbf{y})}{|\mathbf{x}-\mathbf{y}|_{p}^{n-\beta}} d \mathbf{y},
$$

respectively, whenever

$$
\int_{\mathbb{Q}_{p}^{n}}\left|\frac{\Omega\left(|\mathbf{y}|_{p} \mathbf{y}\right) f(\mathbf{y})}{|\mathbf{x}-\mathbf{y}|_{p}^{n-\beta}}\right| d \mathbf{y}<\infty
$$

and

$$
\int_{\mathbb{Q}_{p}^{n}}\left|\frac{b(\mathbf{y}) \Omega\left(|\mathbf{y}|_{p} \mathbf{y}\right) f(\mathbf{y})}{|\mathbf{x}-\mathbf{y}|_{p}^{n-\beta}}\right| d \mathbf{y}<\infty .
$$


In this article, we consider the rough fractional integral operator $T_{\beta, \Omega}^{p}$ along with its commutator $T_{\beta, \Omega}^{p, b}$ and acquire the boundedness on $p$-adic central Morrey spaces. In the latter case, the symbol function is from the $\lambda$-central bounded mean oscillations $\left(C \dot{M} O^{s, \lambda}\right)\left(\mathbb{Q}_{p}^{n}\right)$. The results of the paper can also be implied in locally compact Vilenkin groups and Heisenberg groups. From here on, the letter $C$ means a constant with a different values at separate occurrence.

Definition 1 ([22]). Suppose $1<s<\infty$. and $\lambda \in \mathbb{R}$. The space $\dot{B}^{s, \lambda}\left(\mathbb{Q}_{p}^{n}\right)$ is defined as

$$
\|f\|_{\dot{B} s, \lambda}\left(\mathbb{Q}_{p}^{n}\right)=\sup _{\gamma \in \mathbb{Z}}\left(\frac{1}{\left|B_{\gamma}\right|_{H}^{1+\lambda s}} \int_{B_{\gamma}}|f(\mathbf{x})|^{s} d \mathbf{x}\right)^{1 / s}<\infty,
$$

where $B_{\gamma}=B_{\gamma}(0)$. Moreover, $\dot{B}^{s, \lambda}\left(\mathbb{Q}_{p}^{n}\right)$ reduces to $\{0\}$ for $\lambda<-1 / s$.

Definition 2 ([22]). Suppose $1<s<\infty$ and $\lambda<1 / n$. The space $C M O^{s, \lambda}\left(\mathbb{Q}_{p}^{n}\right)$ is as follows

$$
\|f\|_{C \dot{M} O^{s, \lambda}\left(\mathbb{Q}_{p}^{n}\right)}=\sup _{\gamma \in \mathbb{Z}}\left(\frac{1}{\left|B_{\gamma}\right|_{H}^{1+\lambda s}} \int_{B_{\gamma}}\left|f(\mathbf{x})-f_{B_{\gamma}}\right|^{s} d \mathbf{x}\right)^{1 / s}<\infty,
$$

where $f_{B_{\gamma}}=\frac{1}{\left|B_{\gamma}\right|_{H}} \int_{B_{\gamma}} f(\mathbf{x}) d \mathbf{x}$.

Remark 1. $C M O^{s, \lambda}\left(\mathbb{Q}_{p}^{n}\right)$ is a mere $C M O^{s}\left(\mathbb{Q}_{p}^{n}\right)$ for $\lambda=0$, (see [23]).

Definition 3 ([18]). Suppose $1<s<\infty$ and $\lambda \in \mathbb{R}$. The space $W \dot{B}^{s, \lambda}\left(\mathbb{Q}_{p}^{n}\right)$ is as follows

$$
\|f\|_{W \dot{B}^{s, \lambda}\left(\mathbb{Q}_{p}^{n}\right)}=\sup _{\gamma \in \mathbb{Z}}\left(\frac{\sup _{\sigma>0} \sigma^{s} \mid\left\{\mathbf{x} \in B_{\gamma}:|f(\mathbf{x})|>\left.\sigma\right|_{H}\right.}{\left|B_{\gamma}\right|_{H}^{1+\lambda s}}\right)^{1 / s}<\infty,
$$

where $B_{\gamma}=B_{\gamma}(0)$.

It is noteworthy to illustrate the importance of our main results before stating them. The following example will do a world of good in this context.

Example 1. The solution $u(\mathbf{y}, t)$ of the homogeneous Cauchy problem of linear evolutionary pseudodifferential equation

$$
\left\{\begin{array}{l}
\frac{\partial u}{\partial t}(\mathbf{y}, t)+T_{\beta}^{p} u(\mathbf{y}, t)=0, \quad(\mathbf{y}, t) \in \mathbb{Q}_{p}^{n} \times \mathbb{R}^{+}, \\
u(\mathbf{y}, 0)=u^{0}(\mathbf{y})
\end{array}\right.
$$

is given by $u(\mathbf{y}, t)=\left(T_{\beta}^{p} u^{0}\right)(\mathbf{y})$. For the regularity of the solution, we consider two function spaces $X$ and $Y$. Since $T_{\beta}^{p}$ is linear, then we have

$$
\left\|T_{\beta}^{p}\left(u^{0}-v^{0}\right)\right\|_{Y}=\left\|T_{\beta}^{p}\left(u^{0}\right)-T_{\beta}^{p}\left(v^{0}\right)\right\|_{Y} \leq C\left\|u^{0}-v^{0}\right\|_{X} .
$$

Here, we came across the boundedness inequality

$$
\left\|T_{\beta}^{p} f\right\|_{Y} \leq C\|f\|_{X}
$$

It is imperative to mention here that our operator is very helpful in finding the regularity of Cauchy problem of Schrödinger equation. 


\section{Boundedness of Rough $p$-Adic Fractional Integral Operator on Central Morrey Spaces}

The current section deals the boundednesss of $T_{\beta, \Omega}^{p}$ on central Morrey spaces. However, in order to do this, we need a lemma which can be proved in the same way as [15].

Lemma 1. Suppose $1 \leq q<r<\infty, 0<\beta<n, \beta / n+1 / r=1 / q$, and $\Omega \in L^{q^{\prime}}\left(S_{0}(\mathbf{0})\right)$.

(i) If $f \in L^{q}\left(\mathbb{Q}_{p}^{n}\right), q>1$, then

$$
\left\|T_{\beta, \Omega}^{p} f\right\|_{L^{r}\left(\mathbb{Q}_{p}^{n}\right)} \leq C\|f\|_{L^{q}\left(\mathbb{Q}_{p}^{n}\right)} .
$$

(ii)

$$
\begin{aligned}
& \text { If } f \in L^{1}\left(\mathbb{Q}_{p}^{n}\right), \sigma>0 \text {, then } \\
& \qquad\left|\left\{\mathbf{x} \in \mathbb{Q}_{p}^{n}:\left|T_{\beta, \Omega}^{p} f(\mathbf{x})\right|>\sigma\right\}\right|_{H} \leq C\left(\frac{\|f\|_{L^{1}\left(\mathbb{Q}_{p}^{n}\right)}}{\sigma}\right)^{r} .
\end{aligned}
$$

Now, we turn towards our key result of the section.

Theorem 1. Suppose $0<\beta<n, 1 \leq q<n / \beta, \mu=\beta / n+\lambda, 1 / q-1 / r=\beta / n, \lambda<-\beta / n$, and $\Omega \in L^{q^{\prime}}\left(S_{\mathbf{0}}(\mathbf{0})\right)$.

(i) For $q>1, T_{\beta, \Omega}^{p}$ satisfies the following inequality:

$$
\left\|T_{\beta, \Omega}^{p} f\right\|_{\dot{B}^{r, \mu}\left(\mathbb{Q}_{p}^{n}\right)} \leq C\|f\|_{\dot{B}^{q, \lambda}\left(\mathbb{Q}_{p}^{n}\right)} .
$$

(ii) For $q=1, T_{\beta, \Omega}^{p}$ satisfies the following inequality

$$
\left\|T_{\beta, \Omega}^{p} f\right\|_{W \dot{B}^{r, \mu}\left(\mathbb{Q}_{p}^{n}\right)} \leq C\|f\|_{\dot{B}^{1, \lambda}\left(\mathbb{Q}_{p}^{n}\right)}
$$

Proof. (i) Suppose $f \in \dot{B}^{q, \lambda}\left(\mathbb{Q}_{p}^{n}\right)$. Now for fixed $\gamma \in \mathbb{Z}$, representing $B_{\gamma}(\mathbf{0})$ by $B_{\gamma}$, we begin as

$$
\begin{aligned}
& \left(\frac{1}{\left|B_{\gamma}\right|_{H}^{1+\mu r}} \int_{B_{\gamma}}\left|T_{\beta, \Omega}^{p} f(\mathbf{x})\right|^{r} d \mathbf{x}\right)^{1 / r} \\
& \leq\left(\frac{1}{\left|B_{\gamma}\right|_{H}^{1+\mu r}} \int_{B_{\gamma}}\left|T_{\beta, \Omega}^{p}\left(f \chi_{B_{\gamma}}\right)(\mathbf{x})\right|^{r} d \mathbf{x}\right)^{1 / r} \\
& \quad+\left(\frac{1}{\left|B_{\gamma}\right|_{H}^{1+\mu r}} \int_{B_{\gamma}}\left|T_{\beta, \Omega}^{p}\left(f \chi_{B_{\gamma}^{c}}\right)(\mathbf{x})\right|^{r} d \mathbf{x}\right)^{1 / r} \\
& =I+I I .
\end{aligned}
$$

For $I$, we make use of Lemma 1 together with $\beta / n=1 / q-1 / r$ and $\mu=\lambda+\beta / n$.

$$
\begin{aligned}
I= & \left(\frac{1}{\left|B_{\gamma}\right|_{H}^{1+\mu r}} \int_{B_{\gamma}}\left|T_{\beta, \Omega}^{p}\left(f \chi_{B_{\gamma}}\right)(\mathbf{x})\right|^{r} d \mathbf{x}\right)^{1 / r} \\
& \leq\left|B_{\gamma}\right|_{H}^{-1 / r-\mu}\left(\int_{B_{\gamma}}\left|f \chi_{B_{\gamma}(\mathbf{x})}\right|^{q} d \mathbf{x}\right)^{q} \\
& \leq\|f\|_{\dot{B}^{q, \lambda}\left(\mathbb{Q}_{p}^{n}\right)} .
\end{aligned}
$$

For $I I$, first we have

$$
\int_{S_{k}}\left|\Omega\left(p^{k} \mathbf{y}\right)\right|^{q^{\prime}} d \mathbf{y}=\int_{|\mathbf{x}|_{p}=1}|\Omega(\mathbf{x})|^{q^{\prime}} p^{k n} d \mathbf{x}=C p^{k n}
$$


By the application of Hölder's inequality, equality (11) and $\lambda<-\beta / n$, we proceed as

$$
\begin{aligned}
\left|T_{\beta, \Omega}^{p}\left(f \chi_{B_{\gamma}^{c}}\right)(\mathbf{x})\right| & =\left|\int_{B_{\gamma}^{c}} \frac{\Omega\left(|\mathbf{y}|_{p} \mathbf{y}\right) f(\mathbf{y})}{|\mathbf{x}-\mathbf{y}|_{p}^{n-\beta}} d \mathbf{y}\right| \\
& \leq \int_{B_{\gamma}^{c}} \frac{\left|\Omega\left(|\mathbf{y}|_{p} \mathbf{y}\right) f(\mathbf{y})\right|}{|\mathbf{x}-\mathbf{y}|_{p}^{n-\beta}} d \mathbf{y} \\
& =\sum_{k=\gamma+1}^{\infty} p^{-k(n-\beta)} \int_{S_{k}}\left|\Omega\left(p^{k} \mathbf{y}\right) f(\mathbf{y})\right| d \mathbf{y} \\
& \leq \sum_{k=\gamma+1}^{\infty} p^{-k(n-\beta)}\left(\int_{S_{k}}\left|\Omega\left(p^{k} \mathbf{y}\right)\right|^{q^{\prime}} d \mathbf{y}\right)^{1 / q^{\prime}}\left(\int_{S_{k}}|f(\mathbf{y})|^{q} d \mathbf{y}\right)^{1 / q} \\
& \leq \sum_{k=\gamma+1}^{\infty} p^{-k(n-\beta)}\left(\int_{S_{k}}\left|\Omega\left(p^{k} \mathbf{y}\right)\right|^{q^{\prime}} d \mathbf{y}\right)^{1 / q^{\prime}}\left(\int_{B_{k}}|f(\mathbf{y})|^{q} d \mathbf{y}\right)^{1 / q} \\
& \leq C\|f\|_{\dot{B}^{q, \lambda}\left(\mathbb{Q}_{p}^{n}\right)} \sum_{k=\gamma+1}^{\infty} p^{-k(n-\beta)}\left|B_{k}\right|_{H}^{1+\lambda} \\
& \leq C\left|B_{\gamma}\right|_{H}^{\mu}\|f\|_{\dot{B}^{q, \lambda}\left(\mathbb{Q}_{p}^{n}\right)} .
\end{aligned}
$$

Consequently,

$$
\begin{aligned}
I I & =\left(\frac{1}{\left|B_{\gamma}\right|_{H}^{1+\mu r}} \int_{B_{\gamma}}\left|T_{\beta, \Omega}^{p}\left(f \chi_{B_{\gamma}^{c}}\right)(\mathbf{x})\right|^{r} d \mathbf{x}\right)^{1 / r} \\
& \leq C\|f\|_{\dot{B^{q}}, \lambda\left(\mathbb{Q}_{p}^{n}\right)} .
\end{aligned}
$$

From (10) and (13), we have the desired result.

(ii) For $q=1$ we set $f_{2}=f-f_{1}$ and $f_{1}=f \chi_{B_{\gamma}}$. Then by Lemma 1 , we have

$$
\begin{aligned}
\mid\left\{\mathbf{x} \in B_{\gamma}:\right. & :\left|T_{\beta, \Omega}^{p} f_{1}(\mathbf{x})\right|>\left.\sigma\right|_{H} \\
& \leq C\left(\frac{\left\|f_{1}\right\|_{L^{1}\left(\mathbb{Q}_{p}^{n}\right)}}{\sigma}\right)^{r} \\
& =C \sigma^{-r}\left(\int_{B_{\gamma}}|f(\mathbf{x})| d \mathbf{x}\right)^{r} \\
& \leq C \sigma^{-r}\left|B_{\gamma}\right|_{H}^{(1+\lambda) r}\|f\|_{\dot{B}^{1, \lambda}\left(\mathbb{Q}_{p}^{n}\right)}^{r} \\
& =C \sigma^{-r}\left|B_{\gamma}\right|_{H}^{1+\mu r}\|f\|_{\dot{B}^{1, \lambda}\left(\mathbb{Q}_{p}^{n}\right)}^{r} .
\end{aligned}
$$

Now, from the similar estimate as in (12), we have

$$
\left|T_{\beta, \Omega}^{p} f_{2}(\mathbf{x})\right| \leq C\left|B_{\gamma}\right|_{H}^{\mu}\left\|f_{2}\right\|_{\dot{B}^{1, \lambda}\left(\mathbb{Q}_{p}^{n}\right)}^{r} .
$$

Making use of Chebyshev's inequality, we obtain

$$
\begin{aligned}
\mid\left\{\mathbf{x} \in B_{\gamma}:\left|T_{\beta, \Omega}^{p} f_{2}(\mathbf{x})\right|>\left.\sigma\right|_{H}\right. \\
\leq \sigma^{-r} \int_{B_{\gamma}}\left|T_{\beta, \Omega}^{p} f_{2}(\mathbf{x})\right|^{r} d \mathbf{x} \\
\leq C \sigma^{-r}\left|B_{\gamma}\right|_{H}^{1+\mu r}\left\|f_{2}\right\|_{\dot{B}^{1, \lambda}\left(\mathbb{Q}_{p}^{n}\right)}^{r} \\
\leq C \sigma^{-r}\left|B_{\gamma}\right|_{H}^{1+\mu r}\|f\|_{\dot{B}^{1, \lambda}\left(\mathbb{Q}_{p}^{n}\right)}^{r} .
\end{aligned}
$$


Since

$$
\left|T_{\beta, \Omega}^{p} f(\mathbf{x})\right| \leq\left|T_{\beta, \Omega}^{p} f_{1}(\mathbf{x})\right|+\left|T_{\beta, \Omega}^{p} f_{2}(\mathbf{x})\right|,
$$

we obtain

$$
\begin{aligned}
\mid\left\{\mathbf{x} \in B_{\gamma}:\left|T_{\beta, \Omega}^{p} f(\mathbf{x})\right|>\left.\sigma\right|_{H} \leq\right. & \mid\left\{\mathbf{x} \in B_{\gamma}:\left|T_{\beta, \Omega}^{p} f_{1}(\mathbf{x})\right|>\sigma /\left.2\right|_{H}\right. \\
& +\mid\left\{\mathbf{x} \in B_{\gamma}:\left|T_{\beta, \Omega}^{p} f_{2}(\mathbf{x})\right|>\sigma /\left.2\right|_{H}\right. \\
\leq & C \sigma^{-r}\left|B_{\gamma}\right|_{H}^{1+\mu r}\|f\|_{\dot{B}^{1, \lambda}\left(\mathbb{Q}_{p}^{n}\right)}^{r} .
\end{aligned}
$$

Ultimately,

$$
\left(\frac{\sigma^{r} \mid\left\{\mathbf{x} \in B_{\gamma}:\left|T_{\beta, \Omega}^{p} f(\mathbf{x})\right|>\left.\sigma\right|_{H}\right.}{\left|B_{\gamma}\right|_{H}^{1+\mu r}}\right)^{1 / r} \leq C\left|B_{\gamma}\right|_{H}^{1+\mu r}\|f\|_{\dot{B}^{1, \lambda}\left(\mathbb{Q}_{p}^{n}\right)^{\prime}}^{r}
$$

for some $\gamma \in \mathbb{Z}$ and $\sigma>0$. Hence proof is completed.

\section{3. $\lambda$-Central Bounded Mean Oscillation Estimates of $T_{\beta, \Omega}^{p, b}$ on Central Morrey Spaces}

The following section discusses the $\lambda$-central bounded mean oscillation estimates of $T_{\beta, \Omega}^{p, b}$ on $p$-adic central Morrey spaces. We need an important result before proving this.

Lemma 2 ([22]). Suppose $b \in C \dot{M} O^{r, \lambda}\left(\mathbb{Q}_{p}^{n}\right), \lambda \geq 0$ and $i, j \in \mathbb{Z}$. Then

$$
\left|b_{B_{i}}-b_{B_{j}}\right| \leq p^{n}|i-j|\|b\|_{C \dot{M} O^{r, \lambda}\left(\mathbb{Q}_{p}^{n}\right)} \max \left\{\left|B_{i}\right|_{H}^{\lambda},\left|B_{j}\right|_{H}^{\lambda}\right\}
$$

Now are are firmly in a position to prove our key result.

Theorem 2. Suppose $\beta \in \mathbb{R}, 0<\beta<n, 1<q_{1}<n / \beta, q_{1}^{\prime}<q_{2}<\infty, \beta / n=1 / q_{1}+1 / q_{2}-$ $1 / q$. Let also $0 \leq \lambda_{2}<1 / n, \lambda_{1}+\beta / n<\lambda_{2} \leq 0, \lambda=\lambda_{1}+\lambda_{2}+\beta / n$, and $\Omega \in L^{q_{1}^{\prime}}\left(S_{0}(\mathbf{0})\right)$. Then $T_{\beta, \Omega}^{p, b}$ is bounded from $\dot{B}^{q_{1}, \lambda_{1}}\left(\mathbb{Q}_{p}^{n}\right)$ to $\dot{B}^{q, \lambda}\left(\mathbb{Q}_{p}^{n}\right)$ and satisfies

$$
\left\|T_{\beta, \Omega}^{p, b} f\right\|_{\dot{B}^{q, \lambda}\left(\mathbb{Q}_{p}^{n}\right)} \leq C\|b\|_{C \dot{M} O^{q_{2}, \lambda}\left(\mathbb{Q}_{p}^{n}\right)}\|f\|_{\dot{B}^{q_{1}, \lambda} \lambda_{1}\left(\mathbb{Q}_{p}^{n}\right)} .
$$

Proof. Suppose $f \in \dot{B}^{q, \lambda}\left(\mathbb{Q}_{p}^{n}\right)$. Now for fixed $\gamma \in \mathbb{Z}$, represent $B_{\gamma}(\mathbf{0})$ with $B_{\gamma}$,

$$
\begin{aligned}
& \left(\frac{1}{\left|B_{\gamma}\right|_{H}} \int_{B_{\gamma}}\left|T_{\beta, \Omega}^{p, b} f(\mathbf{x})\right|^{q} d \mathbf{x}\right)^{1 / q} \\
& \leq\left(\frac{1}{\left|B_{\gamma}\right|_{H}} \int_{B_{\gamma}}\left|\left(b(\mathbf{x})-b_{B_{\gamma}}\right)\left(T_{\beta, \Omega}^{p} f \chi_{B_{\gamma}}\right)(\mathbf{x})\right|^{q} d \mathbf{x}\right)^{1 / q} \\
& \quad+\left(\frac{1}{\left|B_{\gamma}\right|_{H}} \int_{B_{\gamma}}\left|\left(b(\mathbf{x})-b_{B_{\gamma}}\right)\left(T_{\beta, \Omega}^{p} f \chi_{B_{\gamma}^{c}}\right)(\mathbf{x})\right|^{q} d \mathbf{x}\right)^{1 / q} \\
& \quad+\left(\frac{1}{\left|B_{\gamma}\right|_{H}} \int_{B_{\gamma}}\left|T_{\beta, \Omega}^{p}\left(\left(b(\mathbf{x})-b_{B_{\gamma}}\right) f \chi_{B_{\gamma}}\right)(\mathbf{x})\right|^{q} d \mathbf{x}\right)^{1 / q} \\
& \quad+\left(\frac{1}{\left|B_{\gamma}\right|_{H}} \int_{B_{\gamma}}\left|T_{\beta, \Omega}^{p}\left(\left(b(\mathbf{x})-b_{B_{\gamma}}\right) f \chi_{B_{\gamma}^{c}}\right)(\mathbf{x})\right|^{q} d \mathbf{x}\right)^{1 / q} \\
& =I_{1}+I_{2}+I_{3}+I_{4} .
\end{aligned}
$$

In order to evaluate $I_{1}$, we set $1 / r=\beta / n-1 / q_{1}, 1 / q=1 / q_{2}+1 / r$, then by Lemma 1 along with Hölder's inequality to have 


$$
\begin{aligned}
& I_{1}=\left(\frac{1}{\left|B_{\gamma}\right|_{H}} \int_{B_{\gamma}}\left|\left(b(\mathbf{x})-b_{B_{\gamma}}\right)\left(T_{\beta, \Omega}^{p} f \chi_{B_{\gamma}}\right)(\mathbf{x})\right|^{q} d \mathbf{x}\right)^{1 / q} \\
& \leq\left|B_{\gamma}\right|_{H}^{-1 / q}\left(\int_{B_{\gamma}}\left|b(\mathbf{x})-b_{B_{\gamma}}\right|^{q_{2}} d \mathbf{x}\right)^{1 / q_{2}} \\
& \cdot\left(\int_{B_{\gamma}}\left|T_{\beta, \Omega}^{p}\left(f \chi_{B_{\gamma}}\right)(\mathbf{x})\right|^{r} d \mathbf{x}\right)^{1 / r} \\
& \leq C\left|B_{\gamma}\right|_{H}^{-1 / r+\lambda_{1}}\|b\|_{C \mathrm{CO}_{2}, \lambda_{2}\left(\mathbb{Q}_{p}^{n}\right)} \\
& \cdot\left(\int_{B_{\gamma}}\left|f \chi_{B_{\gamma}}(\mathbf{x})\right|^{q_{1}} d \mathbf{x}\right)^{1 / q_{1}} \\
& \leq C\left|B_{\gamma}\right|_{H}^{\lambda}\|b\|_{C \dot{M} O^{q_{2}, \lambda_{2}\left(\mathbb{Q}_{p}^{n}\right)}}\|f\|_{\dot{B}_{q_{1}, \lambda_{1}\left(\mathbb{Q}_{p}^{n}\right)}} .
\end{aligned}
$$

In a similar fashion, we estimate $I_{3}$, for this represent $1 / q_{1}+1 / q_{2}=1 / r, \beta / n=1 / r-1 / q$, with Lemma 1; together with Hölder's inequality, we are down to

$$
\begin{aligned}
I_{3}= & \left(\frac{1}{\left|B_{\gamma}\right|_{H}} \int_{B_{\gamma}}\left|T_{\beta_{, \Omega}}^{p}\left(\left(b(\mathbf{x})-b_{B_{\gamma}}\right) f \chi_{B_{\gamma}}\right)(\mathbf{x})\right|^{q} d \mathbf{x}\right)^{1 / q} \\
\leq & C\left|B_{\gamma}\right|_{H}^{-1 / q}\left(\int_{B_{\gamma}}\left|\left(b(\mathbf{x})-b_{B_{\gamma}}\right) f(\mathbf{x})\right|^{r} d \mathbf{x}\right)^{1 / r} \\
\leq & C\left|B_{\gamma}\right|_{H}^{-1 / q}\left(\int_{B_{\gamma}}\left|b(\mathbf{x})-b_{B_{\gamma}}\right|^{q_{2}} d \mathbf{x}\right)^{1 / q_{2}} \\
& \cdot\left(\int_{B_{\gamma}}|f(\mathbf{x})|^{q_{1}} d \mathbf{x}\right)^{1 / q_{1}} \\
\leq & C\left|B_{\gamma}\right|_{H}^{\lambda}\|b\|_{C M O^{q_{2}, \lambda_{2}}\left(\mathbb{Q}_{p}^{n}\right)}\|f\|_{\dot{B}^{q_{1}, \lambda_{1}}\left(\mathbb{Q}_{p}^{n}\right)} .
\end{aligned}
$$

To evaluate $I_{2}$, we use Hölder's inequality, equality (11) and $\lambda_{1}+\beta / n<-\lambda_{2} \leq 0$, we obtain

$$
\begin{aligned}
\left|T_{\beta, \Omega}^{p}\left(f \chi_{B_{\gamma}^{c}}\right)(\mathbf{x})\right| & =\left|\int_{B_{\gamma}^{c}} \frac{\Omega\left(|\mathbf{y}|_{p} \mathbf{y}\right) f(\mathbf{y})}{|\mathbf{x}-\mathbf{y}|_{p}^{n-\beta}} d \mathbf{y}\right| \\
& \leq \int_{B_{\gamma}^{c}} \frac{\left|\Omega\left(|\mathbf{y}|_{p} \mathbf{y}\right) f(\mathbf{y})\right|}{|\mathbf{x}-\mathbf{y}|_{p}^{n-\beta}} d \mathbf{y} \\
& =\sum_{k=\gamma+1}^{\infty} p^{-k(n-\beta)} \int_{S_{k}}\left|\Omega\left(p^{k} \mathbf{y}\right) f(\mathbf{y})\right| d \mathbf{y} \\
& \leq \sum_{k=\gamma+1}^{\infty} p^{-k(n-\beta)}\left(\int_{S_{k}}\left|\Omega\left(p^{k} \mathbf{y}\right)\right|^{q_{1}^{\prime}} d \mathbf{y}\right)^{1 / q_{1}^{\prime}}\left(\int_{S_{k}}|f(\mathbf{y})|^{q_{1}} d \mathbf{y}\right)^{1 / q_{1}} \\
& \leq \sum_{k=\gamma+1}^{\infty} p^{-k(n-\beta)}\left(\int_{S_{k}}\left|\Omega\left(p^{k} \mathbf{y}\right)\right|^{q_{1}^{\prime}} d \mathbf{y}\right)^{1 / q_{1}^{\prime}}\left(\int_{B_{k}}|f(\mathbf{y})|^{q_{1}} d \mathbf{y}\right)^{1 / q_{1}} \\
& \leq C\|f\|_{\dot{B}^{q_{1}, \lambda_{1}}\left(\mathbb{Q}_{p}^{n}\right)} \sum_{k=\gamma+1}^{\infty} p^{-k(n-\beta)}\left|B_{k}\right|_{h}^{1+\lambda_{1}} \\
& =C\|f\|_{\dot{B}^{q_{1}, \lambda_{1}}\left(\mathbb{Q}_{p}^{n}\right)} \sum_{k=\gamma+1}^{\infty} p^{k n\left(\lambda_{1}+\beta / n\right)} \\
& =C\left|B_{\gamma}\right|_{H}^{\lambda_{1}+\beta / n}\|f\|_{\dot{B}^{q_{1}}, \lambda_{1}}\left(\mathbb{Q}_{p}^{n}\right)
\end{aligned}
$$

Now, we are well and truly in a position to estimate $I_{2}$. From (18) and Hölder's inequality, we acquire 


$$
\begin{aligned}
I_{2}= & \left(\frac{1}{\left|B_{\gamma}\right|_{H}} \int_{B_{\gamma}}\left|\left(b(\mathbf{x})-b_{B_{\gamma}}\right)\left(T_{\beta, \Omega}^{p} f \chi_{B_{\gamma}^{c}}\right)(\mathbf{x})\right|^{q} d \mathbf{x}\right)^{1 / q} \\
\leq & C\left|B_{\gamma}\right|_{H}^{\lambda_{1}+\beta / n-1 / q}\|f\|_{\dot{B}^{q_{1}, \lambda_{1}}\left(\mathbb{Q}_{p}^{n}\right)} \\
& \cdot\left(\int_{B_{\gamma}}\left|b(\mathbf{x})-b_{B_{\gamma}}\right|^{q} d \mathbf{x}\right)^{1 / q} \\
\leq & C\left|B_{\gamma}\right|_{H}^{\lambda_{1}+\beta / n-1 / q_{2}}\|f\|_{\dot{B}^{q_{1}, \lambda_{1}}\left(\mathbb{Q}_{p}^{n}\right)} \\
& \cdot\left(\int_{B_{\gamma}}\left|b(\mathbf{x})-b_{B_{\gamma}}\right|^{q_{2}} d \mathbf{x}\right)^{1 / q_{2}} \\
\leq & C\left|B_{\gamma}\right|_{H}^{\lambda}\|b\|_{C \dot{C M O} q_{2}, \lambda_{2}\left(\mathbb{Q}_{p}^{n}\right)}\|f\|_{\dot{B}^{q_{1}, \lambda_{1}}\left(\mathbb{Q}_{p}^{n}\right)} .
\end{aligned}
$$

Finally, we turn our attention towards estimating $I_{4}$. For this we need to give the following estimates. Making use of Hlder's inequality, equality (11), inequality (18), Lemma 2 and of the fact that $\gamma+1 \leq k$, we have

$$
\begin{aligned}
& \left|T_{\beta, \Omega}^{p}\left(\left(b(\mathbf{x})-b_{B_{\gamma}}\right) f \chi_{B_{\gamma}^{c}}\right)(\mathbf{x})\right| \\
& =\left|\int_{B_{\gamma}^{c}} \frac{\left(b(\mathbf{y})-b_{B_{\gamma}}\right) \Omega\left(|\mathbf{y}|_{p} \mathbf{y}\right) f(\mathbf{y})}{|\mathbf{x}-\mathbf{y}|_{p}^{n-\beta}} d \mathbf{y}\right| \\
& \leq \int_{B_{\gamma}^{c}} \frac{\left|b(\mathbf{y})-b_{B_{\gamma}}\right|\left|\Omega\left(|\mathbf{y}|_{p} \mathbf{y}\right) f(\mathbf{y})\right|}{|\mathbf{x}-\mathbf{y}|_{p}^{n-\beta}} d \mathbf{y} \\
& =\sum_{k=\gamma+1}^{\infty} p^{-k(n-\beta)} \int_{S_{k}}\left|b(\mathbf{y})-b_{B_{\gamma}}\right|\left|\Omega\left(p^{k} \mathbf{y}\right) f(\mathbf{y})\right| d \mathbf{y} \\
& \leq \sum_{k=\gamma+1}^{\infty} p^{-k(n-\beta)}\left(\int_{S_{k}}\left|\Omega\left(p^{k} \mathbf{y}\right)\right|^{q_{1}^{\prime}} d \mathbf{y}\right)^{1 / q_{1}^{\prime}} \\
& \cdot\left(\int_{S_{k}}|f(\mathbf{y})|^{q_{1}} d \mathbf{y}\right)^{1 / q_{1}}\left(\int_{S_{k}}\left|b\left(\mathbf{y}-b_{B_{\gamma}}\right)\right|^{q_{2}} d \mathbf{y}\right)^{1 / q_{2}} \\
& \leq \sum_{k=\gamma+1}^{\infty} p^{-k(n-\beta)}\left(\int_{S_{k}}\left|\Omega\left(p^{k} \mathbf{y}\right)\right|^{q_{1}^{\prime}} d \mathbf{y}\right)^{1 / q_{1}^{\prime}} \\
& \cdot\left(\int_{B_{k}}|f(\mathbf{y})|^{q_{1}} d \mathbf{y}\right)^{1 / q_{1}}\left(\int_{B_{k}}\left|b\left(\mathbf{y}-b_{B_{\gamma}}\right)\right|^{q_{2}} d \mathbf{y}\right)^{1 / q_{2}} \\
& \leq C\|f\|_{\dot{B}^{q_{1}, \lambda_{1}}\left(\mathbb{Q}_{p}^{n}\right)} \sum_{k=\gamma+1}^{\infty} p^{-k(n-\beta)}\left|B_{k}\right|_{H}^{1-1 / q_{2}+\lambda_{1}} \\
& \cdot\left(\int_{B_{k}}\left|b\left(\mathbf{y}-b_{B_{\gamma}}\right)\right|^{q_{2}} d \mathbf{y}\right)^{1 / q_{2}} \\
& \leq C\|f\|_{\dot{B}^{q_{1}, \lambda_{1}}\left(\mathbb{Q}_{p}^{n}\right)} \sum_{k=\gamma+1}^{\infty} p^{-k(n-\beta)}\left|B_{k}\right|_{H}^{1-1 / q_{2}+\lambda_{1}} \\
& \left.\cdot\left[\left(\int_{B_{k}}\left|b\left(\mathbf{y}-b_{B_{k}}\right)\right|^{q_{2}} d \mathbf{y}\right)^{1 / q_{2}}+\left.\left(\int_{B_{k}} \mid b_{B_{k}}-b_{B_{\gamma}}\right)\right|^{q_{2}} d \mathbf{y}\right)^{1 / q_{2}}\right] \\
& \leq C\|b\|_{C \dot{M} O_{q_{2},}, \lambda_{2}\left(\mathbb{Q}_{p}^{n}\right)}\|f\|_{\dot{B}^{q_{1}, \lambda_{1}}\left(\mathbb{Q}_{p}^{n}\right)} \sum_{k=\gamma+1}^{\infty} p^{-k(n-\beta)}\left|B_{k}\right|_{H}^{1-1 / q_{2}+\lambda_{1}} \\
& \cdot\left[\left|B_{k}\right|_{H}^{1 / q_{2}+\lambda_{2}}+p^{n}(k-\gamma)\left|B_{k}\right|_{H}^{1 / q_{2}+\lambda_{2}}\right]
\end{aligned}
$$




$$
\begin{aligned}
\leq & C\|b\|_{C \dot{M} O^{q_{2}, \lambda_{2}\left(\mathbb{Q}_{p}^{n}\right)}}\|f\|_{\dot{B}^{q_{1}, \lambda_{1}}\left(\mathbb{Q}_{p}^{n}\right)} \\
& \cdot \sum_{k=\gamma+1}^{\infty}(k-\gamma) p^{-k(n-\beta)}\left|B_{k}\right|_{H}^{1+\lambda_{1}+\lambda_{2}} \\
\leq & C\|b\|_{C \dot{M} O^{q_{2}, \lambda_{2}\left(\mathbb{Q}_{p}^{n}\right)}}\|f\|_{\dot{B}^{q_{1}, \lambda_{1}}\left(\mathbb{Q}_{p}^{n}\right)} \sum_{k=\gamma+1}^{\infty}(k-\gamma) p^{k n \lambda} \\
= & C\left|B_{\gamma}\right|_{H}^{\lambda}\|b\|_{C \dot{M} O_{q_{2}, \lambda_{2}}\left(\mathbb{Q}_{p}^{n}\right)}\|f\|_{\dot{B}^{q_{1}, \lambda_{1}}\left(\mathbb{Q}_{p}^{n}\right)} .
\end{aligned}
$$

Now, it follows from (20) that

$$
\begin{aligned}
& I_{4}=\left(\frac{1}{\left|B_{\gamma}\right|_{H}} \int_{B_{\gamma}}\left|T_{\beta, \Omega}^{p}\left(\left(b(\mathbf{x})-b_{B_{\gamma}}\right) f \chi_{B_{\gamma}^{c}}\right)(\mathbf{x})\right|^{q} d \mathbf{x}\right)^{1 / q} \\
& \leq C\left|B_{\gamma}\right|_{H}^{\lambda}\|b\|_{C M O^{q_{2}, \lambda_{2}}\left(\mathbb{Q}_{p}^{n}\right)}\|f\|_{\dot{B}^{q_{1}, \lambda_{1}}\left(\mathbb{Q}_{p}^{n}\right)} .
\end{aligned}
$$

From (16), (17), (19) and (21), we obtain

$$
\left\|T_{\beta, \Omega}^{p, b} f\right\|_{\dot{B}^{q, \lambda}\left(\mathbb{Q}_{p}^{n}\right)} \leq C\|b\|_{C \dot{M} O^{q_{2}, \lambda_{2}\left(\mathbb{Q}_{p}^{n}\right)}}\|f\|_{\dot{B}_{q_{1}, \lambda_{1}}\left(\mathbb{Q}_{p}^{n}\right)} .
$$

Hence $T_{\beta, \Omega}^{p, b}$ is bounded from $\dot{B}^{q_{1}, \lambda_{1}}\left(\mathbb{Q}_{p}^{n}\right)$ to $\dot{B}^{q, \lambda}\left(\mathbb{Q}_{p}^{n}\right)$. This completes the proof.

\section{Conclusions}

The boundedness of rough $p$-adic fractional integral operator on central Morrey spaces and weak central Morrey spaces in the $p$-adic field is studied. In addition, the boundedness for commutators of rough $p$-adic fractional integral operator on central Morrey spaces is also obtained when the symbol function is from $\lambda$-central bounded mean oscillations. It is noteworthy here that rough $p$-adic fractional integral operator and its commutator can be further considered in locally compact Vilenkin groups, Heisenberg groups, and variable exponent in the $p$-adic field, which will appear elsewhere.

Author Contributions: Conceptualization, N.S.; Data curation, N.S.; Formal analysis, N.S.; Investigation, N.S.; Methodology, N.S. and F.J.; Project administration, F.J.; Validation, F.J.; Visualization, N.S.; supervision, F.J.; Writing—original draft, N.S. and F.J.; Writing—review and editing, N.S. and F.J. All authors have read and agreed to the published version of the manuscript.

Funding: This research received no external funding.

Conflicts of Interest: The authors declare that there have no competing interest.

\section{References}

1. Bohner, M.; Tunç, O.; Tunç, C. Qualitative analysis of caputo fractional integro-differential equations with constant delays. Comp. Appl. Math. 2021, 40, 214. [CrossRef]

2. Abdeljawad, T.; Rashid, S.; Khan, H.; Chu, Y.M. On new fractional integral inequalities for p-convexity within interval-valued functions. Adv. Differ. Equ. 2020, 330, 17. [CrossRef]

3. Mohammed, P.O.; Abdeljawad, T. Integral inequalities for a fractional operator of a function with respect to another function with nonsingular kernel. Adv. Differ. Equ. 2020, 363, 19. [CrossRef]

4. Bosch, P.; Carmenate, H.J.; Rodríguez, J.M.; Sigarreta, J.M. Generalized inequalities involving fractional operators of the RiemannLiouville type. AIMS Math. 2021, 7, 1470-1485. [CrossRef]

5. Grafakos, L. Modern Fourier Analysis, 2nd ed.; Volume 250 of Graduate Texts in Mathematics; Springer: New York, NY, USA, 2008.

6. Stein, M.E. Singular Integrals and Differentiability Properties of Functions; Princeton University Press: Princeton, NJ, USA, 1970.

7. Lacey, M.T.; Moen, K.; Pérez, C.; Torres, R.H. Sharp weighted bounds for fractional integral operators. J. Funct. Anal. 2010, 259, 1073-1097. [CrossRef]

8. Salim, D.; Budhi, W.S.; Soeharyadi, Y. Rough Fractional Integral Operators on Local Morrey Spaces. J. Phys. Conf. Ser. 2019, 1180, 012012. [CrossRef]

9. Wang, H. Estimates for Fractional Integral Operators and Linear Commutators on Certain Weighted Amalgam Spaces. J. Funct. Spaces 2020, 2020, 2697104. [CrossRef] 
10. Kozyrev, S.V. Methods and applications of ultrametric and p-adic analysis: From wavelet theory to biophysics. Proc. Steklov. Inst. Math 2011, 274, 1-84. [CrossRef]

11. Sarfraz, N.; Aslam, M. Some weighted estimates for the commutators of $p$-adic Hardy operator on two weighted $p$-adic Herz-type spaces. AIMS Math. 2021, 6, 9633-9646. [CrossRef]

12. Volosivets, S.S. Maximal function and Reisz potential on $p$-adic linear spaces, $p$-Adic Numbers. Ultrametric Anal. Appl. 2013, 5, 226-234. [CrossRef]

13. Vladimirov, V.S. Tables of integrals of complex Valued Functions of $p$ - Adic Arguments. Proc. Steklov. Inst. Maths. 2014, 284, 1-59. [CrossRef]

14. Vladimirov, V.S.; Volovich, I.V.; Zelenov, E.I. p-Adic Analysis and Mathematical Physics; World Scientific: Singapore, 1994.

15. Taibleson, M.H. Fourier Analysis on Local Fields; Princeton University Press: Princeton, NJ, USA; University of Tokyo Press: Tokyo, Japan, 1975.

16. Haran, S. Riesz potentials and explicit sums in arithmetic. Invent. Math. 1990, 101, 697-703. [CrossRef]

17. Haran, S. Analytic potential theory over the $p$-adics. Ann. Inst. Fourier 1993, 43, 905-944. [CrossRef]

18. Wu, Q.Y.; Mi, L.; Fu, Z.W. Hardy-Littlewood-Sobolev Inequalities on p-adic Central Morrey Spaces. J. Funct. Spaces 2015, 2015, 419532. [CrossRef]

19. Volosivets, S.S. Generalized Fractional Integrals in $p$-Adic Morrey and Herz Spaces, p-Adic Numbers. Ultrametric Anal. Appl. 2017, 9, 53-61. [CrossRef]

20. Ekincioglu, I.; Keskin, C.; Guliyev, R.V. Lipschitz estimates for rough fractional multilinear integral operators on local generalized Morrey spaces. Tbilisi Math. J. 2020, 13, 47-60. [CrossRef]

21. Sarfraz, N.; Aslam, M.; Jarad, F. Boundedness for Commutators of Rough $p$-Adic Hardy Operator on $p$-Adic Central Morrey Spaces. J. Funct. Spaces 2021, 2021, 4886197. [CrossRef]

22. Wu, Q.Y.; Mi, L.; Fu, Z.W. Boundedness of $p$-adic Hardy Operators and their commutators on $p$-adic central Morrey and BMO spaces. J. Funct. Spaces Appl. 2013, 2013, 359193. [CrossRef]

23. Fu, Z.W.; Wu, Q.Y.; Lu, S.Z. Sharp estimates of p-adic Hardy and Hardy-Littlewood-Pólya Operators. Acta Math. Sin. 2013, 29, 137-150. [CrossRef] 